\title{
Potential therapeutic value of dendritic cells loaded with NY-ESO-1 protein for the immunotherapy of advanced hepatocellular carcinoma
}

\author{
YUQING CHEN, AIMIN HUANG, MEIQIN GAO, YONGQIN YAN and WENMIN ZHANG \\ Department of Pathology, Fujian Medical University, Fuzhou, Fujian 350004 P.R. China
}

Received June 20, 2013; Accepted September 17, 2013

DOI: $10.3892 /$ ijmm.2013.1510

\begin{abstract}
NY-ESO-1 is one of the most immunogenic cancertestis (CT) antigens. Cancer vaccine trials based on NY-ESO-1 are currently ongoing. Dendritic cells (DCs) are the most potent antigen-presenting cells. The immune functions of DCs in a number of tumors have been identified; however, the potential therapeutic value of DCs pulsed with NY-ESO-1 in hepatocellular carcinoma (HCC) has not been extensively investigated. The objectives of the present study were to evaluate $\mathrm{T}$ cell response following stimulation with DCs pulsed with the recombinant NY-ESO-1 protein (rESO) and to establish a correlation between NY-ESO-1 expression and clinicopathological features in HCC patients. DCs were generated with granulocyte/macrophage colony-stimulating factor (GM-CSF) and interleukin-4 (IL-4) from human peripheral blood mononuclear cells. A mixed T cell reaction with DCs loaded with recombinant NY-ESO-1 protein (rESO-DCs) was evaluated by MTT assay. $\mathrm{T}$ cell responses against HCC cell lines were analyzed by measuring lactate dehydrogenase (LDH) activity. The protein levels of NY-ESO-1 were detected by immunohistochemistry (IHC) in a tissue microarray (TMA) containing $190 \mathrm{HCC}$ samples. NY-ESO-1 transcript abundance was determined by reverse transcriptase-polymerase chain reaction (RT-PCR) in 54 out of the $190 \mathrm{HCC}$ samples. The results revealed that mature DCs were induced and that rESO-DCs significantly stimulated $\mathrm{T}$ cell proliferation. The specific lysis of $\mathrm{T}$ cells stimulated with rESO-DCs was significantly higher in the NY-ESO-1-positive HCC cells compared with the NY-ESO-1-negative cells and the other controls $(\mathrm{p}<0.01)$. NY-ESO-1 was expressed in $15.8 \%$ (30/190)of the HCC samples, as shown by IHC and in $24.1 \%$ $(13 / 54)$ of the samples, as shown by RT-PCR. The frequency of NY-ESO-1 expression was significantly higher in HCC patients with portal vein tumor thrombosis $(24.6 \%)$ compared with
\end{abstract}

Correspondence to: Professor Wenmin Zhang, Department of Pathology, Fujian Medical University, 88 Jiao Tong Road, Fuzhou, Fujian 350004, P.R. China

E-mail: wminz@163.com

Key words: NY-ESO-1, hepatocellular carcinoma, dendritic cells, immunotherapy those without thrombosis $(11.2 \%, \mathrm{p}=0.013)$. Our data suggest that DCs loaded with NY-ESO-1 protein stimulate antigenspecific T cell responses against HCC cells in vitro. NY-ESO-1 may thus be used as a potential target for immunotherapy in advanced HCC.

\section{Introduction}

Hepatocellular carcinoma (HCC) is the fifth most common type of cancer worldwide and is particularly prevalent in China $(1,2)$. Currently, treatments for HCC include surgical resection, chemotherapy and liver transplantation (3). However, the outcomes remain dismal. The 5-year survival rate for patients with HCC has been reported to be $30-50 \%(4,5)$. Thus, novel, alternative therapeutic options for HCC are urgently required. Immunotherapy for cancer has received much attention in recent years. A number of tumor antigens, such as human telomerase-reverse transcriptase (hTERT) or alphafetoprotein (AFP) have been identified as immunotherapeutic targets, and a number of immunotherapeutic trials have been performed to evaluate the potential therapeutic value of $\mathrm{HCC}$ immunotherapy (6).

Cancer-testis (CT) antigens have been considered attractive targets for cancer immunotherapy due to their restricted expression patterns in a variety of tumors and normal tissues (7). To date, $>150$ genes or gene families encoding $\mathrm{CT}$ antigens have been identified (8). However, only a limited number of CT antigens have been shown to elicit both humoral and cellular responses. NY-ESO-1 (cancer/testis antigen 1B), also known as CTAG1, is one of the most immunogenic CT antigens (9). It was originally found in esophageal cancer by serological recombinant cDNA expression cloning (SEREX) (10) and is expressed in several tumors, including HCC (11-14). NY-ESO-1 expression is associated with a poor tumor outcome and is recognized as a potential biomarker for the prediction of tumor recurrence and treatment outcomes in patients with gastrointestinal stromal tumors and cutaneous melanomas (15).

Due to its expression patterns and strong immunogenicity, NY-ESO-1 has emerged as one of the most attractive targets for cancer vaccines $(7,9)$. The NY-ESO-1 vaccine, based on peptide or protein, has been tested in the treatment of patients with tumors expressing NY-ESO-1, including lung cancer, ovarian cancer, esophageal cancer and melanoma (16-20). The majority 
of the patients showed enhanced immune responses and disease stabilization was achieved in some patients (20). Furthermore, these studies illustrated the safety of various preparations of NY-ESO-1 vaccines. The use of in vitro-generated, autologous dendritic cells (DCs) as a cellular adjuvant for vaccine delivery has been widely tested in cancer patients (21). The potential efficacy of induced immunity against HCC has been supported by a report that the immunization of $2 \mathrm{HCC}$ patients with autologous HCC lysate-loaded DCs resulted in the prolonged survival of $>3$ years in 1 patient (22). However, whether DCs pulsed with NY-ESO-1 protein can induce antigen-specific immune responses against $\mathrm{HCC}$ remains unclear. In addition, the correlation between NY-ESO-1 expression and clinical parameters has not been extensively investigated.

In a previous study, we purified the recombinant NY-ESO-1 protein (rESO) (23). In this study we aimed to evaluate $\mathrm{T}$ cell response against $\mathrm{HCC}$ cell lines following incubation with DCs loaded with rESO. Furthermore, we assessed the mRNA and protein abundance of NY-ESO-1 in HCC samples and determined the correlation between NY-ESO-1 expression and clinical parameters.

\section{Materials and methods}

Patients and samples. A total of 190 paraffin-embedded HCC specimens and their adjacent non-cancerous tissues were collected at the Center for Liver Disease, the First Affiliated Hospital, Fujian Medical University, Fuzhou, China, between 2007 and 2009. Frozen tissues were available in 54 cases. They were frozen immediately in liquid nitrogen after removal from surgical resection and stored at $-80^{\circ} \mathrm{C}$ until use. Informed consent was obtained from all patients. The clinicopathological parameters of these cases are summarized in Table I. Tissue microarrays (TMAs) of the HCC and adjacent non-cancerous liver samples were prepared according to standard procedures (Beecher Instruments Inc., Silver Spring, MD, USA). The study was approved by the Ethical Review Board of Fujian Medical University.

HCC cell lines. The HCC cell lines, H4M and H2P, were kindly provided by Dr JianMing Wen at the Department of Pathology, the First Affiliated Hospital, Sun Yat-sen University, Guangzhou, China. HCC cells were cultured in RPMI-1640 medium with $10 \%$ fetal bovine serum (FBS), L-glutamine, penicillin $(100 \mathrm{IU} / \mathrm{ml})$ and streptomycin $(100 \mu \mathrm{g} / \mathrm{ml})$ at $37^{\circ} \mathrm{C}$.

Preparation of DCs. Peripheral blood mononuclear cells (PBMCs) from healthy volunteers were isolated from blood by Ficoll-Hypaque density gradient centrifugation (Amersham Biosciences, Uppsala, Sweden). The cells were then seeded on 6 -well plates for $2 \mathrm{~h}$ at a density of $2-3 \times 10^{6}$ cells $/ \mathrm{ml}$. Non-adherent cells were removed and adherent cells were incubated in RPMI-1640 medium supplemented with 20\% FBS, $1,000 \mathrm{U} / \mathrm{ml}$ of granulocyte macrophage colony-stimulating factor (GM-CSF) and $500 \mathrm{U} / \mathrm{ml}$ of interleukin-4 (IL-4; PeproTech Inc., Rocky Hill, NJ, USA). After 3 days of incubation, the old medium with floating cells was gently removed and replaced with fresh medium. After 5 days of incubation, $1 / 3$ of the cells were collected as immature DCs (imDCs). The remaining cells were treated with rESO or IL-4 at a concentra-
Table I. Clinicopathological characteristics of HCC patients.

\begin{tabular}{lcc}
\hline Characteristic & $\begin{array}{c}\text { Paraffin-embeded } \\
\text { HCC specimens }\end{array}$ & $\begin{array}{c}\text { Fresh-frozen } \\
\text { HCC specimens }\end{array}$ \\
\hline No. of patients & 190 & 54 \\
Average age (years) & 49 & 50 \\
Age range (years) & $21-75$ & $32-70$ \\
Male/female & $162 / 28$ & $44 / 10$ \\
HBsAg-positive & 155 & 44 \\
AFP ( $\geq 20$ ng/ml) & 132 & 39 \\
With cirrhosis & 172 & 48 \\
With portal vein & 65 & 18 \\
tumor thrombosis & $103 / 87$ & $29 / 25$ \\
$\begin{array}{l}\text { Edmondson's } \\
\text { classification } \\
\text { (grades I-II/III-IV) }\end{array}$ & & \\
\hline
\end{tabular}

HBsAg, hepatitis B surface antigen; AFP, alpha-fetoprotein. HCC, hepatocellular carcinoma.

tion of $50 \mu \mathrm{g} / \mathrm{ml}$ for $24 \mathrm{~h}$. The cells were then incubated with $10 \mathrm{ng} / \mathrm{ml}$ tumor necrosis factor- $\alpha(\mathrm{TNF}-\alpha)$ for $48 \mathrm{~h}$ to induce the formation of mature DCs (mDCs). ImDCs and mDCs were assayed by flow cytometry.

Detection of T cell response. T cells $\left(4 \times 10^{5}\right.$ cells/well) were incubated with imDCs or mDCs at a ratio of $20: 1$ at $37^{\circ} \mathrm{C}$ for $72 \mathrm{~h}$ in RPMI-1640 medium with $20 \% \mathrm{FBS}, 100 \mathrm{U} / \mathrm{ml}$ interleukin-2 (IL-2) and $20 \mu \mathrm{g} / \mathrm{ml}$ phytohaemagglutinin (PHA). Cell proliferation was measured by MTT assay as previously described by Li et al (24). Absorbance was measured at $570 \mathrm{~nm}$ using a Multi-Well Plate Reader (Beckman Coulter Inc., Brea, CA, USA). The proliferation index (PI) of the $\mathrm{T}$ cells was calculated using the following equation: PI = mixed lymphocyte reaction/ lymphocyte reaction. The experiment was conducted 3 times.

Flow cytometry. The level of surface molecules on DCs was determined by flow cytometry using anti-human antibodies: FITC-CD83, PE-CD86 and APC-HLA-DR (BioLegend, San Diego, CA, USA) as previously described (25). Negative controls were fluorochrome-conjugated isotype-matched irrelevant antibodies (Invitrogen, Carlsbad, CA, USA). Briefly, cells suspended in PBS were incubated with antibodies at room temperature for $30 \mathrm{~min}$ in the dark. The cells were then analyzed by a BD FACSCalibur (Becton Dickinson, Franklin Lakes, NJ, USA).

Cytotoxicity assays. Allogeneic T cells were collected as effector cells, and $\mathrm{H} 4 \mathrm{M} / \mathrm{H} 2 \mathrm{P}$ cells were used as the target cells. Effector cells included the rESO-DC-T group (T cells stimulated with DCs loaded with rESO), the IL-4-DC-T group (T cells stimulated with DCs treated with IL-4) and the T cells group (T cells without stimulation). Effector cells and target cells were incubated at effector/target ratios of 20:1 or 50:1 for 
$4 \mathrm{~h}$ at $37^{\circ} \mathrm{C}$ in 96 -well plates. The activity of $\mathrm{T}$ cells against the target tumor cells was measured as previously described in a standard lactate dehydrogenase (LDH) release assay (26). The cytotoxicity of the $\mathrm{T}$ cells was calculated as a percentage of specific lysis using the following formula: \% specific lysis $=($ effector/target release - spontaneous release $) /($ maximal release - spontaneous release) $\mathrm{x} 100 \%$. Data are presented as the means \pm standard deviation.

Immunohistochemistry (IHC). Formalin-fixed slides from TMAs were deparaffinized by xylene and rehydrated by a series of graded alcohol. Endogenous horseradish peroxidase activity was blocked by treatment with $3 \%$ (v/v) $\mathrm{H}_{2} \mathrm{O}_{2}$. Antigen retrieval was achieved by heating the samples in a microwave in $10 \mathrm{mM}$ citrate buffer ( $\mathrm{pH}$ 6.0) for $20 \mathrm{~min}$. Non-specific binding was blocked by incubation with $1 \%(\mathrm{w} / \mathrm{v}) \mathrm{BSA}$ in phosphatebuffered saline (PBS) for $1 \mathrm{~h}$ at room temperature. The slides were then incubated with 1:200 monoclonal anti-NY-ESO-1 antibody (clone E978; Zymed Laboratories, Inc., South San Francisco, CA, USA) overnight at $4^{\circ} \mathrm{C}$. The slides were then incubated with HRP-labeled anti-mouse secondary antibody for $1 \mathrm{~h}$. Immunoreactivity was visualized by diaminobenzidine (DAB). The sections were counterstained with hematoxylin. PBS was used for rinsing between each step. Negative controls were created by omitting the primary antibody. NY-ESO-1 expression was scored by 2 independent observers. The level of NY-ESO-1 expression was described semi-quantitatively using a 4-grade scoring system: -, no staining or focal staining ( $<5 \%$ total);,$+ 5-<25 \% ;++, 25-50 \%$; and,$+++>50 \%$.

Reverse transcriptase-polymerase chain reaction (RT-PCR). Total RNA was extracted from the 54 frozen samples and HCC cell lines using TRIzol reagent (Gibco-BRL, Gaithersburg, MD, USA) according to the manufacturer's instructions. The reverse transcription reaction was performed using the First Strand cDNA Synthesis kit (MBI Fermentas, Vilnius, Lithuania) according to the manufacturer's instructions. Amplification was carried out using the following primers: ESO-1F (exon 1), 5'-cgcctgcttgagttctacctc-3'; and ESO-1R (exon 3), 5'-agggaaagctgctggagacag-3'. The reaction was conducted under the following conditions: $5 \mathrm{~min}$ at $95^{\circ} \mathrm{C}$, followed by $30 \mathrm{sec}$ at $94^{\circ} \mathrm{C}, 1 \mathrm{~min}$ at $60^{\circ} \mathrm{C}$ and $45 \mathrm{sec}$ at $72^{\circ} \mathrm{C}$ for 35 cycles, with a 10 min elongation step at $72^{\circ} \mathrm{C}$. $\beta$-actin was used as the positive control. The expected PCR product sizes of NY-ESO-1 and $\beta$-actin were 219 and 120 bp respectively. Bands were visualized by ethidium bromide staining after separation on a $1.5 \%$ agarose gel. The assay was carried out at least 2 times.

Statistical analysis. Statistical analyses were carried out using SPSS version 13.0 software (SPSS, Chicago, IL, USA). Fisher's exact test or the $\chi^{2}$ test were used to analyze categorical data. Variance analysis was used to determine the statistical significance of the differences between 2 groups. A p-value $<0.05$ was considered to indicate a statistically significant difference.

\section{Results}

$D C$ induction and identification. We obtained $2 \pm 0.31 \times 10^{7}$ mononuclear cells from $50 \mathrm{ml}$ PBMCs following Ficoll sepa-

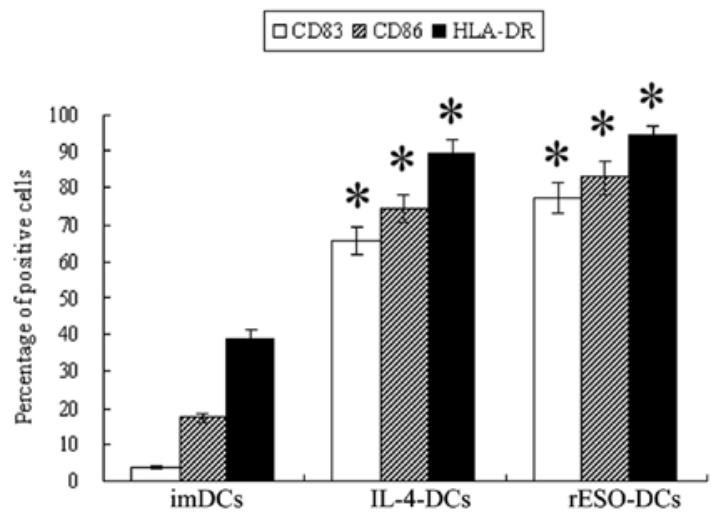

Figure 1. The expression of surface molecules on DCs was assayed by flow cytometry. Data are expressed as the means \pm SD of the percentage positive cells for triplicate assays. The expression of CD83, CD86 and HLA-DR in the rESO-DCs and IL-4-DCs was significantly upregulated compared with the imDC group ( $\left.{ }^{*} \mathrm{p}<0.05\right)$. imDCs, immature dendritic cells (DC), IL-4-DCs, DCs streated with interleukin-4 (IL-4); rESO-DCs, DCs pulsed with recombinant NY-ESO-1 protein (rESO).

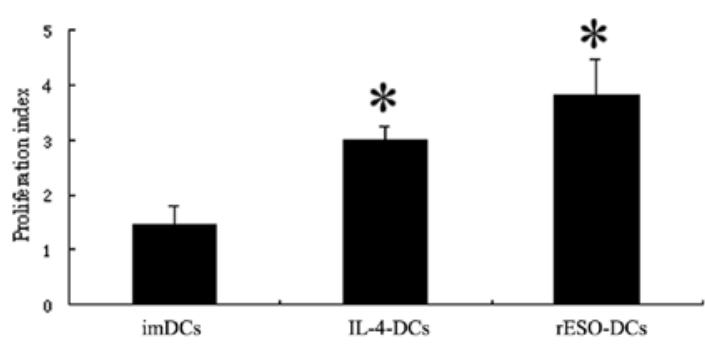

Figure 2. Proliferation index of T lymphocytes following coculture with different dendritic cells (DCs). ${ }^{*} \mathrm{p}<0.05$ vs. imDC group. imDCs, immature DCs; IL-4-DCs, DCs treated with interleukin-4 (IL-4); rESO-DCs, DCs pulsed with recombinant NY-ESO-1 protein (rESO).

ration. The cells were then treated as described in Materials and methods. After 5 days of incubation with GM-CSF and IL-4, 2.2 $\pm 0.49 \times 10^{6}$ dendritic-like cells were obtained based on morphology. Flow cytometry analysis revealed that $38.90 \pm 2.43 \%$ of the imDCs were positive for HLA-DR (Fig. 1). In addition, $3.67 \pm 0.49 \%$ and $17.23 \pm 1.24 \%$ of the imDCs were positive for CD83 and CD86, respectively. After rESO or IL-4 induction for $24 \mathrm{~h}$ and the addition of TNF- $\alpha$ for $48 \mathrm{~h}$, the rESO-DCs or IL-4-DCs showed a typical branch-like appearance. These cells exhibited a significantly higher expression of HLA-DR, CD83 and CD86 (p<0.05) (Fig. 1). Compared with the imDCs, positivity for HLA-DR, CD83 and CD86 increased by 2 - to 21 -fold in the rESO-DCs. Similarly, the percentage of HLA-DR, CD83 and CD86 positivity increased by 2 - to 17 -fold in the IL-4-DCs compared with the imDCs.

To determine the potential of DCs to stimulate $\mathrm{T}$ cell proliferation, we performed a mixed $\mathrm{T}$ lymphocyte reaction by MTT assay. The PI of the T cells mixed with rESO-DCs was higher than that of those mixed with IL-4-DCs and the imDCs $(3.80 \pm 0.66$ vs. $2.99 \pm 0.26$ and $1.44 \pm 0.36)$. A statistically significant difference was observed when comparing the rESO-DCs with the imDCs (p<0.05) (Fig. 2). These data illustrated that DCs pulsed with rESO protein were more effective in stimulating $\mathrm{T}$ lymphocyte proliferation compared with the control cells. 


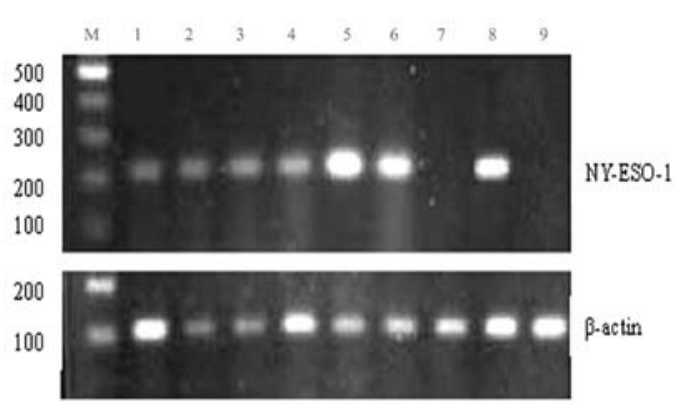

Figure 3. Representative gels of NY-ESO-1 mRNA in hepatocellular carcinoma (HCC) samples and cell lines as determined by reverse transcription-PCR (RT-PCR). The size of the expected product for NY-ESO-1 was 219 bp. HCC tissue (lanes 1-6) and H4M cells (lane 8) were positive for NY-ESO-1 expression. Non-tumorous liver tissue (lane 7) and H2P cells (lane 9) were NY-ESO-1-negative. M, 100 bp molecular weight marker.

Cytotoxicity of NY-ESO-1-specific T lymphocytes. The cytotoxicity of T cells against $2 \mathrm{HCC}$ cell lines was determined by LDH release assay. Allogeneic T cells were collected as the effector cells, and $\mathrm{H} 4 \mathrm{M}$ and $\mathrm{H} 2 \mathrm{P}$ cells were used as the target cells. H4M cells were NY-ESO-1-positive and H2P cells were NY-ESO-1-negative, as shown by RT-PCR (Fig. 3). T cells and HCC cells were incubated at a ratio of $50: 1$ or $20: 1$. When the H4M cells were used as the target cells, the specific NY-ESO-1 $\mathrm{T}$ cells lysed the cancer cells effectively in a dose-dependent manner $(62.13 \pm 5.89 \%$ for ratio $50: 1$ and $49.23 \pm 3.78 \%$ for

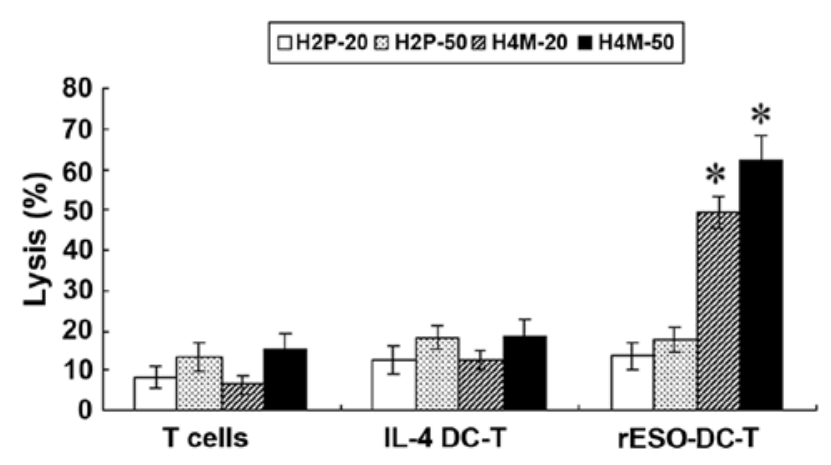

Figure 4. Cytotoxic activity of different $\mathrm{T}$ cells stimulated with dendritic cells (DCs). Following stimulation with DCs [(pulsed with NY-ESO-1 protein or interleukin-4 (IL-4)] or no stimulation, T cells were collected as the effector cells. The HCC cell lines, H4M or H2P, were used as the target cells. Following incubation with effector to target cell (E:T) ratios of 1:20 or 1:50, the cytolytic activity was assessed by measurement of lactate dehydrogenase (LDH) release. Percentage lysis of recombinant NY-ESO-1 protein (rESO)-DC-T against H4M cells was significantly higher than that of the other $\mathrm{T}$ cell groups or against H2P cells ( $\mathrm{p}<0.01)$. rESO-DC-T, T cells stimulated with DCs pulsed with rESO; IL-4-DC-T, T cells stimulated with DCs treated with IL-4; T cells, $\mathrm{T}$ cells without stimulation; H4M-50 or H4M-20, T cells to H4M at ratios of 50:1 or 20:1; H2P-50 or H2P-20, T cells to H2P at ratios of 50:1 or 20:1.

ratio 20:1), significantly higher than that of the IL-4-DC-T and $\mathrm{T}$ cells group $(\mathrm{p}<0.01)$ (Fig. 4). By contrast, the specific lysis among the rESO-DC-T, IL-4-DC-T and T cells did not

Table II. NY-ESO-1 expression in the HCC patients.

\begin{tabular}{|c|c|c|c|c|c|c|}
\hline \multirow[b]{2}{*}{ Group } & \multicolumn{3}{|c|}{$\mathrm{IHC}$} & \multicolumn{3}{|c|}{ RT-PCR } \\
\hline & $\mathrm{N}$ & Positive & $(\%)$ & $\mathrm{N}$ & Positive & $(\%)$ \\
\hline \multicolumn{7}{|l|}{ Gender } \\
\hline Male & 162 & 25 & 15.4 & 44 & 10 & 22.7 \\
\hline Female & 28 & 5 & 17.9 & 10 & 3 & 30.0 \\
\hline \multicolumn{7}{|l|}{ Tumor size } \\
\hline$<5 \mathrm{~cm}$ & 52 & 7 & 13.5 & 13 & 3 & 23.1 \\
\hline$>5 \mathrm{~cm}$ & 138 & 23 & 16.7 & 41 & 10 & 24.4 \\
\hline \multicolumn{7}{|c|}{ Portal vein tumor thrombosis } \\
\hline Positive & 65 & 16 & $24.6^{\mathrm{a}}$ & 18 & 6 & 33.3 \\
\hline Negative & 125 & 14 & 11.2 & 36 & 7 & 19.4 \\
\hline \multicolumn{7}{|c|}{ Edmondson's classification } \\
\hline Grades I-II & 103 & 14 & 13.6 & 29 & 6 & 20.7 \\
\hline Grades III- IV & 87 & 16 & 18.4 & 25 & 7 & 28 \\
\hline \multicolumn{7}{|l|}{ HBsAg } \\
\hline Positive & 155 & 24 & 15.5 & 44 & 10 & 22.7 \\
\hline Negative & 35 & 6 & 17.1 & 10 & 3 & 30.0 \\
\hline \multicolumn{7}{|l|}{$\mathrm{AFP}$} \\
\hline$<20 \mathrm{ng} / \mathrm{ml}$ & 58 & 9 & 15.5 & 15 & 3 & 20.0 \\
\hline$\geq 20 \mathrm{ng} / \mathrm{ml}$ & 132 & 21 & 15.9 & 39 & 10 & 25.6 \\
\hline Total & 190 & 30 & 15.8 & 54 & 13 & 24.1 \\
\hline
\end{tabular}

${ }^{a} \mathrm{p}<0.05$ compared with HCC patients without portal vein tumor thrombosis. HCC, hepatocellular carcinoma; IHC, immunohistochemistry; AFP, alpha-fetoprotein. 

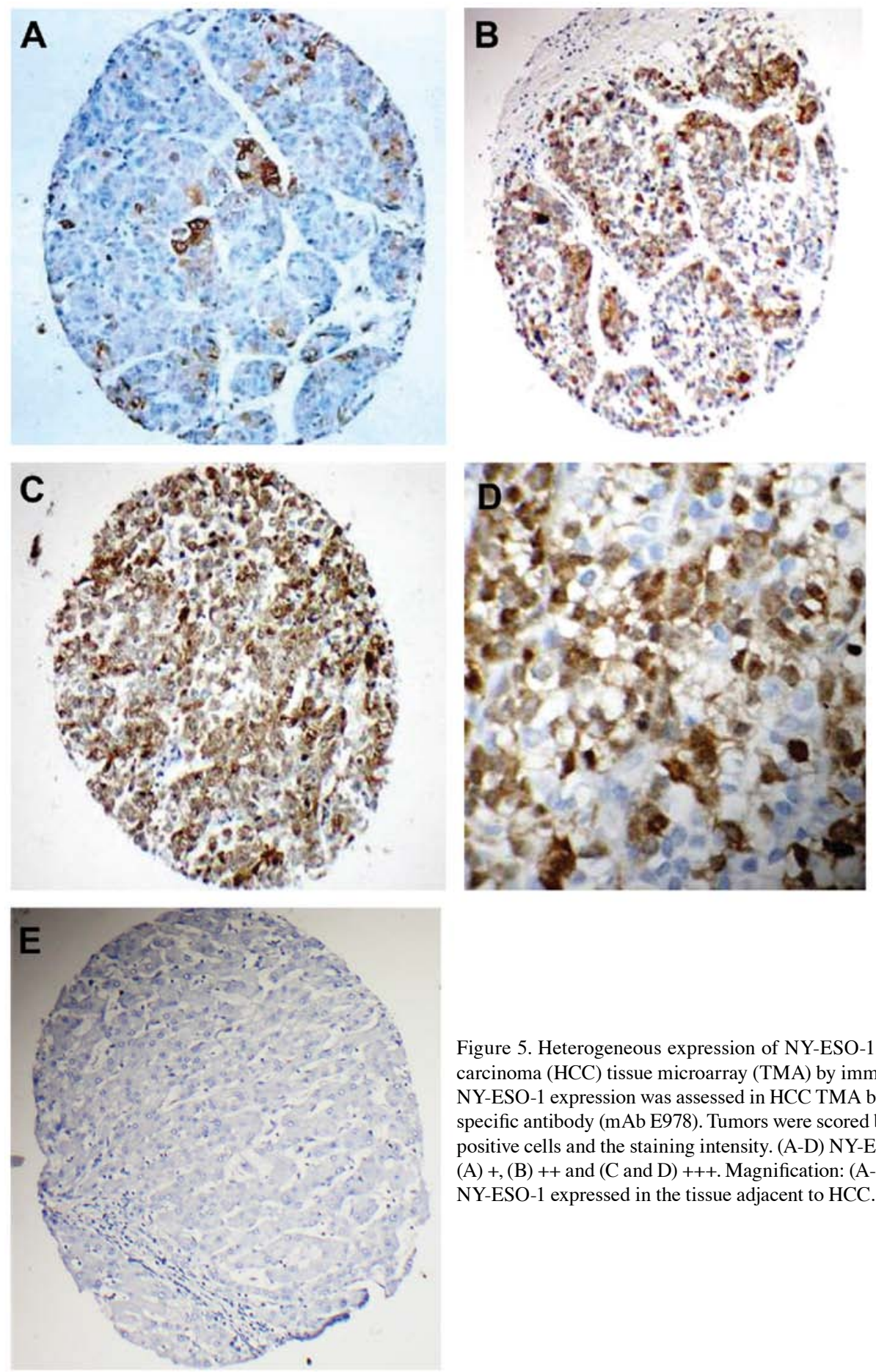

Figure 5. Heterogeneous expression of NY-ESO-1 protein in hepatocellular carcinoma (HCC) tissue microarray (TMA) by immunohistochemistry (IHC). NY-ESO-1 expression was assessed in HCC TMA by IHC using the NY-ESOspecific antibody (mAb E978). Tumors were scored based on the percentage of positive cells and the staining intensity. (A-D) NY-ESO-1 expression scored as (A) +, (B) ++ and (C and D) +++. Magnification: (A-C) x100; (D) x400. (E) No NY-ESO-1 expressed in the tissue adjacent to HCC. Magnification, x100.

differ significantly in the H2P cells, which did not express NY-ESO-1. These results demonstrate that T cells stimulated with DCs pulsed with rESO exert significant antigen-specific lysis on HCC cells which express NY-ESO-1.

NY-ESO-1 expression in HCC patients. IHC analyses of NY-ESO-1 protein indicated that a total of 30 out of the 190 HCC specimens expressed NY-ESO-1 (15.8\%). Among these, $8(4.2 \%)$ were graded as,$+++ 12(6.3 \%)$ as ++ , and $10(5.3 \%)$ as + (Fig. 5A-C). NY-ESO-1 was located predominantly in the cytoplasm, although nuclear staining was observed in a few cells (Fig. 5D). No staining was observed in the tissue adjacent to HCC (Fig. 5E). We also determined the expression profile of NY-ESO-1 mRNA in 54 tumors by RT-PCR. A detectable NY-ESO-1 transcript was observed in 10 tumors (18.5\%). Representative results are shown in Fig. 3.

Correlation between NY-ESO-1 expression and clinical parameters. Table II summarizes the correlation between NY-ESO-1 expression and the clinicopathological characteristics of the tumor samples. In the HCC patients with portal vein tumor thrombosis, the frequency of NY-ESO-1 positivity was $24.6 \%(16 / 65)$. By contrast, the frequency of NY-ESO-1 positivity in the HCC patients without portal vein tumor thrombosis 
Table III. Expression of NY-ESO-1 in 54 HCC samples detected by IHC and RT-PCR.

\begin{tabular}{lccccc}
\hline & \multicolumn{5}{c}{ IHC } \\
\cline { 2 - 6 } RT-PCR & $+(\mathrm{n}=5)$ & $++(\mathrm{n}=2)$ & $+++(\mathrm{n}=3)$ & Negative $(\mathrm{n}=44)$ & Total \\
\hline Positive & 4 & 2 & 3 & 4 & 13 \\
Negative & 1 & 0 & 0 & 40 & 41 \\
Total & 5 & 2 & 3 & 44 & 54 \\
\hline
\end{tabular}

HCC, hepatocellular carcinoma; IHC, immunohistochemistry; RT-PCR, reverse transcriptase-polymerase chain reaction.

was $11.2 \%$ (14/125), which was significantly lower compared with the HCC patients with portal vein tumor thrombosis $(\mathrm{p}=0.013)$. Statistical analysis also revealed that the frequency of the detectable NY-ESO-1 transcript was higher in HCC patients with portal vein tumor thrombosis as compared with the HCC patients without portal vein tumor thrombosis (33.3 vs. $19.4 \%, \mathrm{p}=0.21$ ). Statistical analysis did not reveal a correlation between NY-ESO-1 expression and age, gender, tumor size, histological grade or hepatitis B surface antigen (HBsAg)/ AFP status in the HCC samples.

Correlation between NY-ESO-1 mRNA and protein levels in HCC patients. A total of $54 \mathrm{HCC}$ samples were examined for both the transcript and protein levels of NY-ESO-1. A total of 10 cases were positive for NY-ESO-1 protein, as shown by by IHC and 13 tumors expressed NY-ESO-1 mRNA, as shown by RT-PCR. As presented in Table III, 4 cases were positive for NY-ESO-1 mRNA expression, as shown by RT-PCR, but were shown to be negative by IHC. Out of the 10 tumors with positive immunostaining, 1 tumor was shown to be negative by RT-PCR. This case displayed positive IHC staining for NY-ESO-1.

\section{Discussion}

The survival of HCC patients is poor despite advancements in HCC therapy (3). In recent years, immunotherapy has become a promising strategy for tumor therapy. A variety of immunotherapy regimens have emerged for HCC patients, including HCC lysates (27), tumor cell-DC fusion (28) and cytokines (29). However, the effects of these methods have been limited. The major barrier to antigen-specific immunotherapy in $\mathrm{HCC}$ is a lack of well-defined immunogenic tumor antigens. It has been shown that NY-ESO-1 is one of the most immunogenic CT antigens (7). Immune responses against NY-ESO-1 have been induced in a number of tumors, such as melanoma (30), ovarian cancer (31) and lung cancer (12). The NY-ESO-1 vaccine has been investigated in clinical trials of melanoma and ovarian cancer (32). Recently, a NY-ESO-1 vaccine has also been examined in esophageal and prostate cancer patients (33). Patients bearing NY-ESO-1-expressing tumors displayed an effective induction of NY-ESO-1 antibody and CD4/CD8 $\mathrm{T}$ cell responses. Although only a few studies have reported the immune responses induced by NY-ESO-1 in HCC patients (34), NY-ESO-1-specific immune responses in HCC are not yet well understood. In our study, our results demonstrated that
NY-ESO-1-specific T cell responses were induced, which significantly increased the lysis of NY-ESO-1-expressing HCC cells in vitro. These data provide evidence supporting the use of NY-ESO-1-based immunotherapy and suggest that NY-ESO-1 may be a useful target for the immunotherapy of HCC patients.

For cancer clinical trials targeted against defined antigens, a detailed knowledge of the antigen expression is crucial. Our data indicated that the positive rate of NY-ESO-1 protein was $15.8 \%$ and the NY-ESO-1 mRNA expression was $24.1 \%$ in the HCC samples, which was higher than that found in the study by Luo et al (35), and is comparable to the results of Wang et al (13). We also illustrated a high concordance between RT-PCR and IHC for NY-ESO-1 expression in the HCC samples. The HCC patients displayed moderate or high levels of NY-ESO-1 and as shown by IHC, they were positive for the NY-ESO-1 transcript. However, we detected positivity for the NY-ESO-1 transcript in 4 HCC samples without a detectable NY-ESO-1 protein expression. A likely explanation for this is that RT-PCR has a higher sensitivity to detect NY-ESO-1 than IHC. In this study, we also observed heterogeneity for NY-ESO-1 expression by IHC in a few cases, and this information could not be achieved by RT-PCR. It may therefore be desirable to use both IHC and RT-PCR to obtain information regarding the expression level and antigen distribution.

NY-ESO-1 has been speculated to be a prognostic marker in gastrointestinal stromal tumors, melanoma and HCC $(15,36,37)$. NY-ESO-1 expression is associated with a worse tumor outcome. The present study also revealed that NY-ESO-1 expression in HCC patients with portal vein tumor thrombosis had a significantly higher intensity and positivity compared with that in HCC patients without portal vein tumor thrombosis, as shown by IHC and RT-PCR. Thus, it can be hypothesized that the NY-ESO-1 gene may play an important role in tumor invasion and progression. Although the mechanisms involved are unclear, a NY-ESO-1 vaccine may play an important role in the advanced stages of disease, as also found in esophageal carcinoma by Bujas et al (38).

In conclusion, we identified specific $\mathrm{T}$ cell responses stimulated with DCs pulsed with NY-ESO-1 in HCC cell lines. We also demonstrate that NY-ESO-1 is heterogeneously expressed in HCC patients, specifically in advanced HCC patients with portal vein tumor thrombosis. This suggests that a DC-based NY-ESO-1 vaccine may prove to be effective for immunotherapy in advanced HCC. Moreover, a combination of demethylation or preparation of multiple $\mathrm{CT}$ antigens may increase the efficacy of HCC immunotherapy. 


\section{Acknowledgements}

This study was supported by the Natural Science Foundation of Fujian Province (C0610023, 2012J01362). We are grateful to Dr JianMing Wen (Department of Pathology, the First Affiliated Hospital, Sun Yat-sen University) for providing the HCC cell lines in this study. We also thank Kay Ka-Wai Li. (Department of Anatomical and Cellular Pathology, the Chinese University of Hong Kong) for revising this article.

\section{References}

1. El-Serag HB and Rudolph KL: Hepatocellular carcinoma: epidemiology and molecular carcinogenesis. Gastroenterology 132 2557-2576, 2007.

2. Chen MS, Peng ZW, Xu L, Zhang YJ, Liang HH and Li JQ: Role of radiofrequency ablation in the treatment of hepatocellular carcinoma: experience of a cancer center in China. Oncology 81 (Suppl 1): S100-S104, 2011.

3. Rossi L, Zoratto F, Papa A, et al: Current approach in the treatment of hepatocellular carcinoma. World J Gastrointest Oncol 2: 348-359, 2010.

4. Shimozawa $\mathrm{N}$ and Hanazaki K: Longterm prognosis after hepatic resection for small hepatocellular carcinoma. J Am Coll Surg 198: 356-365, 2004.

5. Chang $\mathrm{CH}$, Chau GY, Lui WY, Tsay SH, King KL and Wu CW: Long-term results of hepatic resection for hepatocellular carcinoma originating from the noncirrhotic liver. Arch Surg 139: 320-326, 2004.

6. Greten TF, Manns MP and Korangy F: Immunotherapy of HCC. (Review). Rev Recent Clin Trials 3: 31-39, 2008.

7. Caballero OL and Chen YT: Cancer/testis (CT) antigens: potential targets for immunotherapy. Cancer Sci 100: 2014-2021, 2009.

8. Hofmann O, Caballero OL, Stevenson BJ, et al: Genome-wide analysis of cancer/testis gene expression. Proc Natl Acad Sci USA 105: 20422-20427, 2008.

9. Gnjatic S, Nishikawa H, Jungbluth AA, et al: NY-ESO-1: review of an immunogenic tumor antigen. Adv Cancer Res 95: 1-30, 2006.

10. Chen YT, Scanlan MJ, Sahin U, et al: A testicular antigen aberrantly expressed in human cancers detected by autologous antibody screening. Proc Natl Acad Sci USA 94: 1914-1918, 1997.

11. Oba-Shinjo SM, Caballero OL, Jungbluth AA, et al: Cancer-testis (CT) antigen expression in medulloblastoma. Cancer Immun 8: 7, 2008.

12. Kim SH, Lee $\mathrm{S}$, Lee $\mathrm{CH}$, et al: Expression of cancer-testis antigens MAGE-A3/6 and NY-ESO-1 in non-small-cell lung carcinomas and their relationship with immune cell infiltration. Lung 187: 401-411, 2009.

13. Wang XY, Chen HS, Luo S, Zhang HH, Fei R and Cai J: Comparisons for detecting NY-ESO-1 mRNA expression levels in hepatocellular carcinoma tissues. Oncol Rep 21: 713-719, 2009.

14. Grigoriadis A, Caballero OL, Hoek KS, et al: CT-X antigen expression in human breast cancer. Proc Natl Acad Sci USA 106: 13493-13498, 2009.

15. Svobodová S, Browning J, MacGregor D, et al: Cancer-testis antigen expression in primary cutaneous melanoma has independent prognostic value comparable to that of Breslow thickness, ulceration and mitotic rate. Eur J Cancer 47: 460-469, 2011.

16. Jäger $\mathrm{E}$, Karbach J, Gnjatic S, et al: Recombinant vaccinia/ fowlpox NY-ESO-1 vaccines induce both humoral and cellular NY-ESO-1-specific immune responses in cancer patients. Proc Natl Acad Sci USA 103: 14453-14458, 2006.

17. Bender A, Karbach J, Neumann A, et al: LUD 00-009: phase 1 study of intensive course immunization with NY-ESO-1 peptides in HLA-A2 positive patients with NY-ESO-1-expressing cancer. Cancer Immun 7: 16, 2007.

18. Odunsi K, Qian F, Matsuzaki J, et al: Vaccination with an NY-ESO-1 peptide of HLA class I/II specificities induces integrated humoral and T cell responses in ovarian cancer. Proc Natl Acad Sci USA 104: 12837-12842, 2007.
19. Nicholaou T, Ebert L, Davis ID, et al: Directions in the immune targeting of cancer: lessons learned from the cancer-testis $\mathrm{Ag}$ NY-ESO-1. Immunol Cell Biol 84: 303-317, 2006.

20. Kakimi K, Isobe M, Uenaka A, et al: A phase I study of vaccination with NY-ESO-1f peptide mixed with Picibanil OK-432 and Montanide ISA-51 in patients with cancers expressing the NY-ESO-1 antigen. Int J Cancer 129: 2836-2846, 2011.

21. Engell-Noerregaard L, Hansen TH, Andersen MH, Thor Straten P and Svane IM: Review of clinical studies on dendritic cell-based vaccination of patients with malignant melanoma: assessment of correlation between clinical response and vaccine parameters. Cancer Immunol Immunother 58: 1-14, 2009.

22. Ladhams A, Schmidt C, Sing G, et al: Treatment of non-resectable hepatocellular carcinoma with autologous tumor-pulsed dendritic cells. J Gastroenterol Hepatol 17: 889-896, 2002.

23. Zhang W, Xiao G, Zhang M, Xie D, Guo A and Wen J: The prokaryotic expression, purification and preliminary application of human NY-ESO-1 gene. Zhongguo Kang Ai Xie Hui 32: 626-629, 2005 (In Chinese).

24. Li DY, Gu C, Min J, Chu ZH and Ou QJ: Maturation induction of human peripheral blood mononuclear cell-derived dendritic cells. Exp Ther Med 4: 131-134, 2012.

25. Della Bella S, Nicola S, Riva A, Biasin M,Clerici M and Villa ML: Functional repertoire of dendritic cells generated in granulocyte macrophage-colony stimulating factor and interferon-alpha. J Leukoc Biol 75: 106-116, 2004.

26. Wang XH, Qin Y, Hu MH and Xie Y: Dendritic cells pulsed with gp96-peptide complexes derived from human hepatocellular carcinoma (HCC) induce specific cytotoxic T lymphocytes. Cancer Immunol Immunother 54: 971-980, 2005.

27. Pan K, Zhao JJ, Wang H, et al: Comparative analysis of cytotoxic $\mathrm{T}$ lymphocyte response induced by dendritic cells loaded with hepatocellular carcinoma-derived RNA or cell lysate. Int J Biol Sci 6: 639-648, 2010.

28. Cao DY, Yang JY, Yue SQ, et al: Comparative analysis of DC fused with allogeneic hepatocellular carcinoma cell line HepG2 and autologous tumor cells as potential cancer vaccines against hepatocellular carcinoma. Cell Immunol 259: 13-20, 2009.

29. Rinaldi M, Iurescia S, Fioretti D, Ponzetto A and Carloni G: Strategies for successful vaccination against hepatocellular carcinoma. Int J Immunopathol Pharmacol 22: 269-277, 2009.

30. Gedye C, Quirk J, Browning J, et al: Cancer/testis antigens can be immunological targets in clonogenic $\mathrm{CD} 133^{+}$melanoma cells. Cancer Immunol Immunother 58: 1635-1646, 2009.

31. Milne K, Barnes RO, Girardin A, et al: Tumor-infiltrating T cells correlate with NY-ESO-1-specific autoantibodies in ovarian cancer. PLoS One 3: e3409, 2008.

32. Odunsi K, Matsuzaki J, Karbach J, et al: Efficacy of vaccination with recombinant vaccinia and fowlpox vectors expressing NY-ESO-1 antigen in ovarian cancer and melanoma patients. Proc Natl Acad Sci USA 109: 5797-5802, 2012.

33. Kawada J, Wada $\mathrm{H}$, Isobe M, et al: Heteroclitic serological response in esophageal and prostate cancer patients after NY-ESO-1 protein vaccination. Int J Cancer 130: 584-592, 2012.

34. Korangy F, Ormandy LA, Bleck JS, et al: Spontaneous tumorspecific humoral and cellular immune responses to NY-ESO-1 in hepatocellular carcinoma. Clin Cancer Res 10: 4332-4341, 2004.

35. Luo G, Huang S, Xie X, et al: Expression of cancer-testis genes in human hepatocellular carcinomas. Cancer Immun 2: 11, 2002.

36. Perez D, Hauswirth F, Jäger D, et al: Protein expression of cancer testis antigens predicts tumor recurrence and treatment response to imatinib in gastrointestinal stromal tumors. Int J Cancer 128: 2947-2952, 2011.

37. Xu H, Gu N, Liu ZB, et al: NY-ESO-1 expression in hepatocellular carcinoma: A potential new marker for early recurrence after surgery. Oncol Lett 3: 39-44, 2012.

38.Bujas T, Marusic Z, Peric Balja M, Mijic A, Kruslin B and Tomas D: MAGE-A3/4 and NY-ESO-1 antigens expression in metastatic esophageal squamous cell carcinoma. Eur J Histochem 55: e7, 2011. 